\title{
LISA optimal sensitivity
}

\author{
Thomas A. Prince, ${ }^{*}$ Massimo Tinto, ${ }^{\dagger}$ and Shane L. Larson ${ }^{\ddagger}$ \\ Space Radiation Laboratory, California Institute of Technology, Pasadena, California 91125 \\ J. W. Armstrong ${ }^{\S}$ \\ Jet Propulsion Laboratory, California Institute of Technology, Pasadena, California 91109
}

(Received 9 September 2002; published 17 December 2002)

\begin{abstract}
The multiple Doppler readouts available on the Laser Interferometer Space Antenna (LISA) permit simultaneous formation of several interferometric observables. All these observables are independent of laser frequency fluctuations and have different couplings to gravitational waves and to the various LISA instrumental noises. Within the functional space of interferometric combinations LISA will be able to synthesize, we have identified a triplet of interferometric combinations that show optimally combined sensitivity. As an application of the method, we computed the sensitivity improvement for sinusoidal sources in the nominal, equal-arm LISA configuration. In the part of the Fourier band where the period of the wave is longer than the typical light travel-time across LISA, the sensitivity gain over a single Michelson interferometer is equal to $\sqrt{2}$. In the mid-band region, where the LISA Michelson combination has its best sensitivity, the improvement over the Michelson sensitivity is slightly better than $\sqrt{2}$, and the frequency band of best sensitivity is broadened. For frequencies greater than the reciprocal of the light travel-time, the sensitivity improvement is oscillatory and $\sim \sqrt{3}$, but can be greater than $\sqrt{3}$ near frequencies that are integer multiples of the inverse of the one-way light travel-time in the LISA arm.
\end{abstract}

DOI: 10.1103/PhysRevD.66.122002

PACS number(s): 04.80.Nn, 07.60.Ly, 95.55.Ym

\section{INTRODUCTION}

The Laser Interferometer Space Antenna (LISA) is a deep-space mission, jointly proposed to the National Aeronautics and Space Administration (NASA) and the European Space Agency (ESA), to detect and study gravitational radiation in the millihertz frequency band.

LISA will use coherent laser beams exchanged between three remote, widely separated, spacecraft. Modeling each spacecraft as carrying lasers, beam splitters, photo-detectors, and drag-free proof masses on each of two optical benches, it has been shown $[1-3]$ that the six measured time series of Doppler shifts of the one-way laser beams between spacecraft pairs, and the six measured shifts between adjacent optical benches on each spacecraft, can be combined, with suitable time delays, to cancel the otherwise overwhelming frequency fluctuations of the lasers $\left(\Delta \nu / \nu \simeq 10^{-13} / \sqrt{\mathrm{Hz}}\right)$, and the noise due to the mechanical vibrations of the optical benches (which could be as large as $\Delta \nu / \nu \simeq 10^{-16} / \sqrt{\mathrm{Hz}}$ ). The achievable strain sensitivity level $h \simeq 10^{-21} / \sqrt{\mathrm{Hz}}$ is set by the buffeting of the drag-free proof masses inside each optical bench, and by the shot noise at the photodetectors.

In contrast to Earth-based, equi-arm interferometers for gravitational wave detection, LISA will have multiple read-

\footnotetext{
*Also at Jet Propulsion Laboratory, California Institute of Technology, Pasadena, CA 91109. Electronic address: prince@ srl.caltech.edu

${ }^{\dagger}$ Also at Jet Propulsion Laboratory, California Institute of Technology, Pasadena, CA 91109. Electronic address: Massimo.Tinto@jpl.nasa.gov

\#Electronic address: shane@ srl.caltech.edu

${ }^{\S}$ Electronic address: John.W.Armstrong@jpl.nasa.gov
}

outs. The data they generate, when properly time shifted and linearly combined, provide observables that are not only insensitive to laser frequency fluctuations and optical bench motions, but also show different couplings to gravitational radiation and to the remaining system noises $[1,2,4]$. The space of all possible interferometric combinations can be generated by properly combining four generators [1], and it has been shown to be an algebraic module [5]. In this paper we derive from first principles a particular combination of these generators which, for a given waveform and source location in the sky, give maximum signal-to-noise ratio. In this respect, LISA should no longer be regarded as a single detector, but rather as an array of interferometers working in coincidence.

An outline of the paper is presented here. Section II provides a brief summary of Time-Delay Interferometry, the data processing technique needed to remove the frequency fluctuations of the six lasers used by LISA. After showing that the entire set of interferometric combinations can be derived by properly combining four generators, $(\alpha, \beta, \gamma, \zeta)$, in Sec. III we turn to the problem of optimization of the signal-to-noise ratio (SNR) within this functional space. As an application, we apply our results to the case of sinusoidal signals randomly polarized and randomly distributed on the celestial sphere. We find that the standard LISA sensitivity figure derived for a single Michelson Interferometer [2] can be improved by a factor of $\sqrt{2}$ in the low-part of the frequency band, and by more than $\sqrt{3}$ in the remaining part of the accessible band. In Sec. IV we present our comments and conclusions.

\section{TIME-DELAY INTERFEROMETRY FOR LISA}

Figure 1 shows the overall LISA geometry. The spacecraft are labeled 1,2, 3 and distances between pairs of spacecraft 


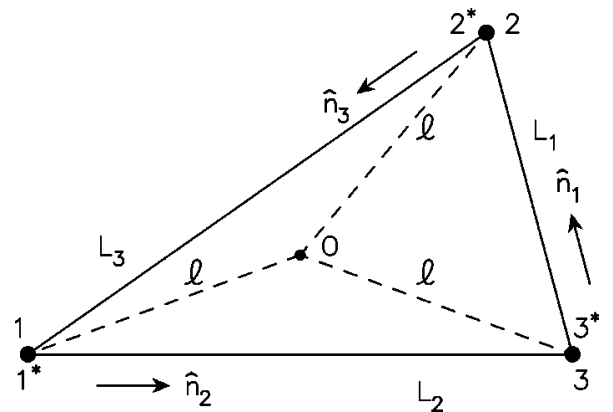

FIG. 1. Schematic LISA configuration. Each spacecraft is equidistant from the point $\mathrm{O}$, in the plane of the spacecraft. Unit vectors $\hat{n}_{i}$ point between spacecraft pairs with the indicated orientation. At each vertex spacecraft there are two optical benches (denoted 1, $1^{*}$, etc.), as indicated.

are $L_{1}, L_{2}, L_{3}$, with $L_{i}$ being opposite spacecraft $i$. Unit vectors between spacecraft are $\hat{n}_{i}$, oriented as indicated in Fig. 1. We similarly index the relative frequency fluctuations $y_{i j}$ data to be analyzed: $y_{31}$ is the relative frequency fluctuations time series measured at reception at spacecraft 1 with transmission from spacecraft 2 (along $L_{3}$ ). Similarly, $y_{21}$ is the corresponding time series derived from reception at spacecraft 1 with transmission from spacecraft 3 . The other four one-way relative frequency time series from signals exchanged between the spacecraft are obtained by cyclic permutation of the indices: $1 \rightarrow 2 \rightarrow 3 \rightarrow 1$. The useful notation for delayed data streams will also be used: $y_{31,2}=y_{31}(t$ $\left.-L_{2}\right), y_{31,23}=y_{31}\left(t-L_{2}-L_{3}\right)=y_{31,32}$, etc. (units in which $c=1)$. Six more Doppler difference series result from laser beams exchanged between adjacent optical benches within each spacecraft; these are similarly indexed as $z_{i j}(i, j$ $=1,2,3 ; i \neq j$ ) (see [2] and [3] for details).

The light paths for the $y_{i 1}$ 's and $z_{i 1}$ 's can be traced in Fig. 2. An outgoing light beam transmitted to a distant spacecraft is routed from the laser on the local optical bench using mirrors and beam splitters; this beam does not interact with the local proof mass. Conversely, an incoming light beam from a distant spacecraft is bounced off the local proof mass before being reflected onto the photodetector where it is mixed with light from the laser on that same optical bench. These data are denoted $y_{31}$ and $y_{21}$ in Fig. 2. Beams exchanged between adjacent optical benches however do precisely the opposite. Light to be transmitted from the laser on an optical bench is first bounced off the proof mass it encloses and then directed via fiber optics to the other optical bench. Upon reception it does not interact with the proof mass there, but is directly mixed with local laser light. They are $z_{31}$ and $z_{21}$ in Fig. 2 .

The frequency fluctuations introduced by the gravitational wave signal, the lasers, the optical benches, the proof masses, the fiber optics, and the measurement itself at the photodetector (shot noise) enter into the Doppler observables $y_{i j}, z_{i j}$ with specific time signatures. They have been derived in the literature $[2,3]$, and we refer the reader to those papers for a detailed discussion. The Doppler data $y_{i j}, z_{i j}$ are the fundamental measurements needed to synthesize all the in-

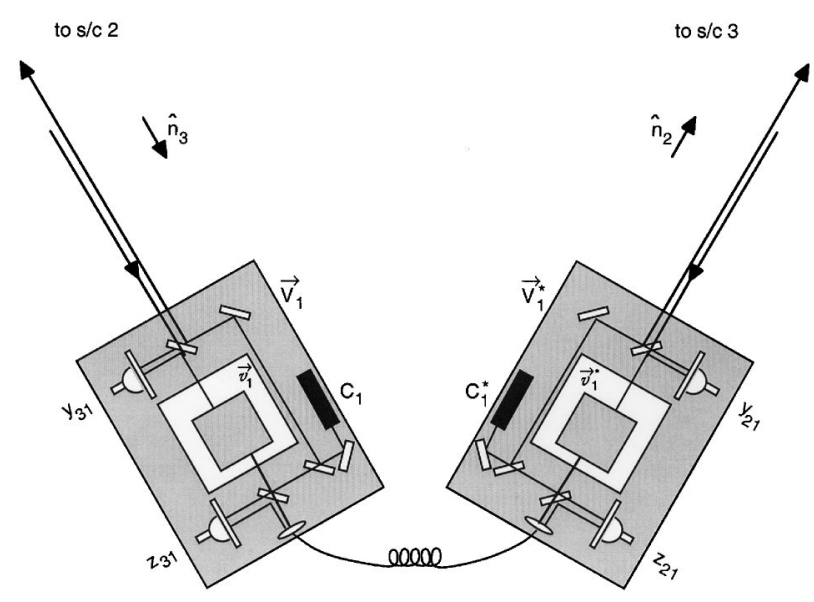

FIG. 2. Schematic diagram of proof-masses-plus-opticalbenches for a LISA spacecraft. The left-hand bench reads out the Doppler signals $y_{31}$ and $z_{31}$. The right hand bench analogously reads out $y_{21}$ and $z_{21}$. The random velocities of the two proof masses and two optical benches are indicated (lower case $\vec{v}_{i}$ for the proof masses, upper case $\vec{V}_{i}$ for the optical benches).

terferometric observables unaffected by laser and optical bench noises.

The simplest such combination, the totally symmetrized Sagnac response $\zeta$, uses all the data of Fig. 2 symmetrically,

$$
\begin{aligned}
\zeta= & y_{32,2}-y_{23,3}+y_{13,3}-y_{31,1}+y_{21,1}-y_{12,2} \\
& +\frac{1}{2}\left(-z_{13,21}+z_{23,12}-z_{21,23}+z_{31,23}-z_{32,13}+z_{12,13}\right) \\
& +\frac{1}{2}\left(-z_{32,2}+z_{12,2}-z_{13,3}+z_{23,3}-z_{21,1}+z_{31,1}\right)
\end{aligned}
$$

and its transfer functions to gravitational waves and instrumental noises were derived in [1], and [2] respectively. In particular, $\zeta$ has a "six-pulse response" to gravitational radiation, i.e. a $\delta$-function gravitational wave signal produces six distinct pulses in $\zeta[1]$, which are located with relative times depending on the arrival direction of the wave and the detector configuration.

Together with $\zeta$, three more interferometric combinations, $(\alpha, \beta, \gamma)$, jointly generate the entire space of interferometric combinations $[1,2,5]$. Their expressions in terms of the measurements $y_{i j}, z_{i j}$ are as follows:

$$
\begin{aligned}
\alpha= & y_{21}-y_{31}+y_{13,2}-y_{12,3}+y_{32,12}-y_{23,13} \\
& -\frac{1}{2}\left(z_{13,2}+z_{13,13}+z_{21}+z_{21,123}+z_{32,3}+z_{32,12}\right) \\
& +\frac{1}{2}\left(z_{23,2}+z_{23,13}+z_{31}+z_{31,123}+z_{12,3}+z_{12,12}\right),
\end{aligned}
$$

with $\beta$ and $\gamma$ derived by permuting the spacecraft indices in $\alpha$. As in the case of $\zeta$, a $\delta$-function gravitational wave produces six pulses in $\alpha, \beta$, and $\gamma$. 
We should remind the reader that the four interferometric responses $(\alpha, \beta, \gamma, \zeta)$ satisfy the following relationship:

$$
\zeta-\zeta_{, 123}=\alpha_{, 1}-\alpha_{, 23}+\beta_{, 2}-\beta_{, 13}+\gamma_{, 3}-\gamma_{, 12} \text {. }
$$

Jointly they also give the expressions of the interferometric combinations derived in [1,2]: the Unequal-arm Michelson $(\mathrm{X}, \mathrm{Y}, \mathrm{Z})$, the Beacon $(\mathrm{P}, \mathrm{Q}, \mathrm{R})$, the Monitor $(\mathrm{E}, \mathrm{F}, \mathrm{G})$, and the Relay $(\mathrm{U}, \mathrm{V}, \mathrm{W})$ responses

$$
\begin{aligned}
\mathrm{X}_{, 1} & =\alpha_{, 23}-\beta_{, 2}-\gamma_{, 3}+\zeta, \\
\mathrm{P} & =\zeta-\alpha_{, 1}, \\
\mathrm{E} & =\alpha-\zeta_{, 1}, \\
\mathrm{U} & =\gamma_{, 1}-\beta,
\end{aligned}
$$

with the remaining expressions obtained from Eqs. (4)-(7) by permutation of the spacecraft indices. All these interferometric combinations have been shown to add robustness to the mission with respect to failures of subsystems, and potential design, implementation, or cost advantages $[1,2]$.

\section{OPTIMAL SENSITIVITY FOR LISA}

All the above interferometric combinations have been shown to individually have rather different sensitivities [2], as a consequence of their different responses to gravitational radiation and system noises. LISA has the capability of simultaneously observing a gravitational wave signal with many different interferometric combinations, all having different antenna patterns and noises. We should thus no longer regard LISA as a single detector system but rather as an array of gravitational wave detectors working in coincidence. This suggests that the presently adopted LISA sensitivity could be improved by optimally combining the four generators $(\alpha, \beta, \gamma, \zeta)$. In mathematical terms this can be restated by saying that we should be able to find that particular combination of the four generators that has a maximum signal-tonoise ratio to a given gravitational wave signal.

In order to proceed with this idea, let us consider the following linear combination $\eta(f)$ of the Fourier transforms of the four generators $(\tilde{\alpha}, \widetilde{\beta}, \widetilde{\gamma}, \widetilde{\zeta})$ :

$$
\begin{aligned}
\eta(f) \equiv & a_{1}(f, \vec{\lambda}) \tilde{\alpha}(f)+a_{2}(f, \vec{\lambda}) \widetilde{\beta}(f) \\
& +a_{3}(f, \vec{\lambda}) \tilde{\gamma}(f)+a_{4}(f, \vec{\lambda}) \widetilde{\zeta}(f),
\end{aligned}
$$

where the $\left\{a_{i}(f, \vec{\lambda})\right\}_{i=1}^{4}$ are arbitrary complex functions of the Fourier frequency $f$, and of a vector $\vec{\lambda}$ containing parameters characterizing the gravitational wave signal (source location in the sky, waveform parameters, etc.) and the noises affecting the four responses (noise levels, their correlations, etc.). For a given choice of the four functions $\left\{a_{i}\right\}_{i=1}^{4}, \eta$ gives an element of the functional space of interferometric combinations generated by $(\alpha, \beta, \gamma, \zeta)$. Our goal is therefore to identify, for a given gravitational wave signal, the four functions $\left\{a_{i}\right\}_{i=1}^{4}$ that maximize the signal-to-noise ratio, $S N R_{\eta}^{2}$, of the combination $\eta$

$$
S N R_{\eta}^{2}=\int_{f_{l}}^{f_{u}} \frac{\left|a_{1} \tilde{\alpha}_{s}+a_{2} \widetilde{\beta}_{s}+a_{3} \tilde{\gamma}_{s}+a_{4} \widetilde{\zeta}_{s}\right|^{2}}{\left\langle\left|a_{1} \tilde{\alpha}_{n}+a_{2} \widetilde{\beta}_{n}+a_{3} \tilde{\gamma}_{n}+a_{4} \widetilde{\zeta}_{n}\right|^{2}\right\rangle} d f
$$

In Eq. (9) the subscripts $s$ and $n$ refer to the signal and the noise parts of $(\tilde{\alpha}, \widetilde{\beta}, \widetilde{\gamma}, \widetilde{\zeta})$ respectively, the angle brackets represent noise ensemble averages, and the interval of integration $\left(f_{l}, f_{u}\right)$ corresponds to the frequency band accessible by LISA.

Before proceeding with the maximization of the $S N R_{\eta}^{2}$ we may notice from Eq. (3) that the Fourier transform of the totally symmetric Sagnac combination, $\widetilde{\zeta}$, multiplied by the transfer function $1-e^{2 \pi i f\left(L_{1}+L_{2}+L_{3}\right)}$ can be written as a linear combination of the Fourier transforms of the remaining three generators $(\tilde{\alpha}, \widetilde{\beta}, \tilde{\gamma})$. Since the signal-to-noise ratios of $\eta$ and $\left(1-e^{2 \pi i f\left(L_{1}+L_{2}+L_{3}\right)}\right) \eta$ are equal, we may conclude that the optimization of the signal-to-noise ratio of $\eta$ can be performed only on the three observables $\alpha, \beta, \gamma$. This implies the following redefined expression for $S N R_{\eta}^{2}$ :

$$
S N R_{\eta}^{2}=\int_{f_{l}}^{f_{u}} \frac{\left|a_{1} \tilde{\alpha}_{s}+a_{2} \widetilde{\beta}_{s}+a_{3} \tilde{\gamma}_{s}\right|^{2}}{\left\langle\left|a_{1} \tilde{\alpha}_{n}+a_{2} \widetilde{\beta}_{n}+a_{3} \tilde{\gamma}_{n}\right|^{2}\right\rangle} d f .
$$

The $S N R_{\eta}^{2}$ can be regarded as a functional over the space of the three complex functions $\left\{a_{i}\right\}_{i=1}^{3}$, and the particular set of complex functions that extremize it can of course be derived by solving the associated set of Euler-Lagrange equations.

In order to make the derivation of the optimal SNR easier, let us first denote by $\mathbf{x}^{(s)}$ and $\mathbf{x}^{(n)}$ the two vectors of the signals $\left(\widetilde{\alpha}_{s}, \widetilde{\beta}_{s}, \widetilde{\gamma}_{s}\right)$ and the noises $\left(\widetilde{\alpha}_{n}, \widetilde{\beta}_{n}, \widetilde{\gamma}_{n}\right)$ respectively. Let us also define a to be the vector of the three functions $\left\{a_{i}\right\}_{i=1}^{3}$, and denote with $\mathbf{C}$ the Hermitian, nonsingular, correlation matrix of the vector random process $\mathbf{x}_{n}$

$$
(\mathbf{C})_{r t} \equiv\left\langle\mathbf{x}_{r}^{(n)} \mathbf{x}_{t}^{(n) *}\right\rangle \text {. }
$$

If we finally define $(\mathbf{A})_{i j}$ to be the components of the Hermitian matrix $\mathbf{x}_{i}^{(s)} \mathbf{x}_{j}^{(s) *}$, we can rewrite $S N R_{\eta}^{2}$ in the following form:

$$
S N R_{\eta}^{2}=\int_{f_{l}}^{f_{u}} \frac{\mathbf{a}_{i} \mathbf{A}_{i j} \mathbf{a}_{j}^{*}}{\mathbf{a}_{r} \mathbf{C}_{r t} \mathbf{a}_{t}^{*}} d f
$$

where we have adopted the usual convention of summation over repeated indices. Since the noise correlation matrix $\mathbf{C}$ is nonsingular, and the integrand is positive definite or null, the stationary values of the signal-to-noise ratio will be attained at the stationary values of the integrand, which are given by solving the following set of equations (and their complex conjugated expressions):

$$
\frac{\partial}{\partial \mathbf{a}_{k}}\left[\frac{\mathbf{a}_{i} \mathbf{A}_{i j} \mathbf{a}_{j}^{*}}{\mathbf{a}_{r} \mathbf{C}_{r t} \mathbf{a}_{t}^{*}}\right]=0, \quad k=1,2,3 .
$$

After taking the partial derivatives, Eq. (13) can be rewritten in the following form: 


$$
\left(\mathbf{C}^{-1}\right)_{i r}(\mathbf{A})_{r j}\left(\mathbf{a}^{*}\right)_{j}=\left[\frac{\mathbf{a}_{p} \mathbf{A}_{p q} \mathbf{a}_{q}^{*}}{\mathbf{a}_{l} \mathbf{C}_{l m} \mathbf{a}_{m}^{*}}\right]\left(\mathbf{a}^{*}\right)_{i}, \quad i=1,2,3
$$

which tells us that the stationary values of the signal-to-noise ratio of $\eta$ are equal to the eigenvalues of the matrix $\mathbf{C}^{-1}$ -A. The result in Eq. (13) is well known in the theory of quadratic forms, and it is called Rayleigh's principle [7,8].

In order now to identify the eigenvalues of the matrix $\mathbf{C}^{-1} \cdot \mathbf{A}$, we first notice that the $3 \times 3$ matrix $\mathbf{A}$ has rank 1 . This implies that the matrix $\mathbf{C}^{-1} \cdot \mathbf{A}$ has also rank 1 , as it is easy to verify. Therefore two of its three eigenvalues are equal to zero, while the remaining nonzero eigenvalue represents the solution we are looking for.

The analytic expression of the third eigenvalue can be obtained by using the property that the trace of the $3 \times 3$ matrix $\mathbf{C}^{-1} \cdot \mathbf{A}$ is equal to the sum of its three eigenvalues, and in our case to the eigenvalue we are looking for. From these considerations we derive the following expression for the optimized signal-to-noise ratio $S N R_{\eta \text { opt }}^{2}$ :

$$
S N R_{\eta \mathrm{opt}}^{2}=\int_{f_{l}}^{f_{u}} \mathbf{x}_{i}^{(s)} *\left(\mathbf{C}^{-1}\right)_{i j} \mathbf{x}_{j}^{(s)} d f
$$

We can summarize the results derived in this section, which are given by Eqs. (10), (15), in the following way:

(i) Among all possible interferometric combinations LISA will be able to synthesize with its four generators $\alpha, \beta, \gamma, \zeta$, the particular combination giving maximum signal-to-noise ratio can be obtained by using only three of them, namely $(\alpha, \beta, \gamma)$.

(ii) The expression of the optimal signal-to-noise ratio given by Eq. (15) implies that LISA should be regarded as a network of three interferometer detectors of gravitational radiation [of responses $(\alpha, \beta, \gamma)]$ working in coincidence [9].

\section{Application}

As an application of Eq. (15), here we calculate the sensitivity that LISA can reach when observing sinusoidal signals uniformly distributed on the celestial sphere and of random polarization. In order to calculate the optimal signal-tonoise ratio we will also need to use a specific expression for the noise correlation matrix $\mathbf{C}$. As a simplification, we will assume the LISA arm-lengths to be equal to its nominal value $L=16.67 \mathrm{sec}$, the optical-path noises to be equal and uncorrelated to each other, and finally the noises due to the proof-mass noises to be also equal, uncorrelated to each other and to the optical-path noises. Under these assumptions the correlation matrix becomes real, its three diagonal elements are equal, and all the off-diagonal terms are equal to each other, as it is easy to verify by direct calculation [2]. The noise correlation matrix $\mathbf{C}$ is therefore uniquely identified by two real functions, $S_{\alpha}$ and $S_{\alpha \beta}$, in the following way:

$$
\mathbf{C}=\left(\begin{array}{ccc}
S_{\alpha} & S_{\alpha \beta} & S_{\alpha \beta} \\
S_{\alpha \beta} & S_{\alpha} & S_{\alpha \beta} \\
S_{\alpha \beta} & S_{\alpha \beta} & S_{\alpha}
\end{array}\right) .
$$

The expression of the optimal signal-to-noise ratio assumes a rather simple form if we diagonalize this correlation matrix by properly "choosing a new basis." There exists an orthogonal transformation of the generators $(\tilde{\alpha}, \widetilde{\beta}, \tilde{\gamma})$ which will transform the optimal signal-to-noise ratio into the sum of the signal-to-noise ratios of the "transformed" three interferometric combinations. The expressions of the three eigenvalues $\left\{\mu_{i}\right\}_{i=1}^{3}$ (which are real) of the noise correlation matrix $\mathbf{C}$ can easily be found by using the algebraic manipulator MATHEMATICA [10], and they are equal to

$$
\mu_{1}=\mu_{2}=S_{\alpha}-S_{\alpha \beta}, \quad \mu_{3}=S_{\alpha}+2 S_{\alpha \beta} .
$$

Note that two of the three real eigenvalues, $\left(\mu_{1}, \mu_{2}\right)$, are equal. This implies that the eigenvector associated to $\mu_{3}$ is orthogonal to the two-dimensional space generated by the eigenvalue $\mu_{1}$, while any chosen pair of eigenvectors corresponding to $\mu_{1}$ will not necessarily be orthogonal. This inconvenience can be avoided by choosing an arbitrary set of vectors in this two-dimensional space, and by orthonormalizing them. After some simple algebra, we have derived the following three ortho-normalized eigenvectors:

$$
\begin{aligned}
& \mathbf{v}_{1}=\frac{1}{\sqrt{2}}(-1,0,1), \\
& \mathbf{v}_{2}=\frac{1}{\sqrt{6}}(1,-2,1), \\
& \mathbf{v}_{3}=\frac{1}{\sqrt{3}}(1,1,1) .
\end{aligned}
$$

Equation (17) implies the following three linear combinations of the generators $(\tilde{\alpha}, \widetilde{\beta}, \tilde{\gamma})$ :

$$
\begin{aligned}
& A=\frac{1}{\sqrt{2}}(\tilde{\gamma}-\tilde{\alpha}), \\
& E=\frac{1}{\sqrt{6}}(\tilde{\alpha}-2 \widetilde{\beta}+\tilde{\gamma}), \\
& T=\frac{1}{\sqrt{3}}(\tilde{\alpha}+\widetilde{\beta}+\tilde{\gamma}),
\end{aligned}
$$

where $A, E$, and $T$ are italicized to indicate that these are "orthogonal modes." Although the expressions for the modes $A$ and $E$ depend on our particular choice for the two eigenvectors $\left(\mathbf{v}_{1}, \mathbf{v}_{2}\right)$, it is clear from our earlier considerations that the value of the optimal signal-to-noise ratio is unaffected by such a choice. From Eq. (18) it is also easy to verify that the noise correlation matrix of these three combinations is diagonal, and that its nonzero elements are indeed equal to the eigenvalues given in Eq. (16).

In order to calculate the sensitivity corresponding to the expression of the optimal signal-to-noise ratio, we have proceeded similarly to what was done in $[1,2]$, and described in 
more detail in [11]. We assume an equal-arm LISA ( $L$ $=16.67$ light seconds), and take the one-sided spectra of proof mass and aggregate optical-path-noises (on a single link), expressed as fractional frequency fluctuation spectra, to be ( $[2,6]), S_{y}^{\text {proof mass }}=2.5 \times 10^{-48}[\mathrm{f} / 1 \mathrm{~Hz}]^{-2} \mathrm{~Hz}^{-1}$ and $S_{y}^{\text {opticalpath }}=1.8 \times 10^{-37}[\mathrm{f} / 1 \mathrm{~Hz}]^{2} \mathrm{~Hz}^{-1}$, respectively. We also assume that aggregate optical path noise has the same transfer function as shot noise.

The optimum SNR is the square root of the sum of the squares of the SNRs of the three "orthogonal modes" $(A, E, T)$. To compare with previous sensitivity curves of a single LISA Michelson interferometer, we construct the SNRs as a function of Fourier frequency for sinusoidal waves from sources uniformly distributed on the celestial sphere. To produce the SNR of each of the $(A, E, T)$ modes we need the gravitational wave response and the noise response as a function of Fourier frequency. We build up the gravitational wave responses of the three modes $(A, E, T)$ from the gravitational wave responses of $(\alpha, \beta, \gamma)$. For 7000 Fourier frequencies in the $\sim 10^{-4} \mathrm{~Hz}$ to $\sim 1 \mathrm{~Hz}$ LISA band, we produce the Fourier transforms of the gravitational wave response of $(\alpha, \beta, \gamma)$ from the formulas in $[1,11]$. The averaging over source directions (uniformly distributed on the celestial sphere) and polarization states (uniformly distributed on the Poincare sphere) is performed via a Monte Carlo method. From the Fourier transforms of the $(\alpha, \beta, \gamma)$ responses at each frequency, we construct the Fourier transforms of $(A, E, T)$. We then square and average to compute the mean-squared responses of $(A, E, T)$ at that frequency from $10^{4}$ realizations of (source position, polarization state) pairs.

The noise spectra of $(A, E, T)$ are determined from the raw spectra of proof-mass and optical-path noises, and the transfer functions of these noises to $(A, E, T)$. Using the transfer functions given in [2], the resulting spectra are equal to

$$
\begin{aligned}
& S_{A}(f)=S_{E}(f)=16 \sin ^{2}(\pi f L)[3+2 \cos (2 \pi f L)+\cos (4 \pi f L)] S_{y}^{\text {proof mass }}(f)+8 \sin ^{2}(\pi f L)[2+\cos (2 \pi f L)] S_{y}^{\text {optical path }}(f), \\
& S_{T}(f)=\left[2+4 \cos ^{2}(2 \pi f L)\right]\left[4 \sin ^{2}(\pi f L) S_{y}^{\text {proof mass }}+S_{y}^{\text {optical path }}(f)\right] .
\end{aligned}
$$

Let the amplitude of the sinusoidal gravitational wave be $h$. The SNR for, e.g. $A, S N R_{A}$, at each frequency $f$ is equal to $h$ times the ratio of the root-mean-squared gravitational wave response at that frequency divided by $\sqrt{S_{A}(f) B}$, where $B$ is the bandwidth conventionally taken to be equal to 1 cycle per year. Finally, if we take the reciprocal of $S N R_{A} / h$ and multiply it by 5 to get the conventional $S N R=5$ sensitivity criterion, we obtain the sensitivity curve for this combination which can then be compared against the corresponding sensitivity curve for the equal-arm Michelson Interferometer.

In Fig. 3 we show the sensitivity curve for the LISA equal-arm Michelson response $(S N R=5)$ as a function of the Fourier frequency, and the sensitivity curve from the optimum weighting of the data described above: $5 h / \sqrt{S N R_{A}^{2}+S N R_{E}^{2}+S N R_{T}^{2}}$. The SNRs were computed for a bandwidth of 1 cycle/year. Note that at frequencies where the LISA Michelson combination has best sensitivity, the improvement in signal-to-noise ratio provided by the optimal combination is slightly larger than $\sqrt{2}$.

In Fig. 4 we plot the ratio between the optimal SNR and the SNR of a single Michelson interferometer. In the longwavelength limit, the SNR improvement is $\sqrt{2}$. For Fourier frequencies greater than or about equal to $1 / L$, the SNR improvement is larger and varies with the frequency, showing an average value of about $\sqrt{3}$. In particular, for bands of frequencies centered on integer multiples of $1 / L, S N R_{T}$ contributes strongly and the aggregate SNR in these bands can be greater than 2 . In order to better understand the contribution from the three different combinations to the optimal combination of the three generators, in Fig. 5 we plot the signal-to-noise ratios of $(A, E, T)$ as well as the optimal signal-to-noise ratio. For an assumed $h=10^{-23}$, the SNRs of the three modes are plotted versus frequency. For the equalarm case computed here, the SNRs of $A$ and $E$ are equal across the band. In the long wavelength region of the band, modes $A$ and $E$ have SNRs much greater than mode $T$, where its contribution to the total SNR is negligible. At higher frequencies, however, the $T$ combination has SNR greater than

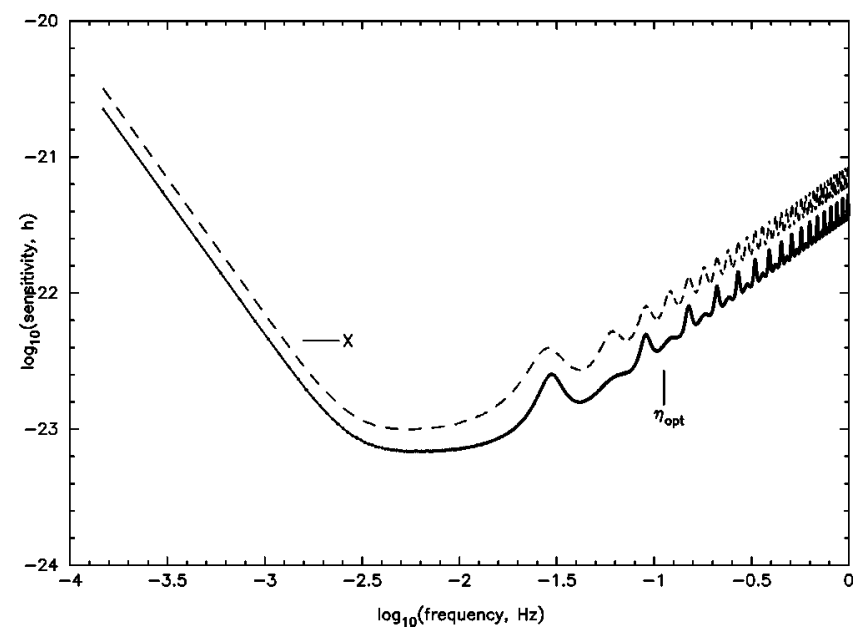

FIG. 3. The LISA Michelson sensitivity curve $(\mathrm{SNR}=5)$ and the sensitivity curve for the optimal combination of the data, both as a function of Fourier frequency. The integration time is equal to one year, and LISA is assumed to have a nominal arm length $L$ $=16.67 \mathrm{sec}$. 


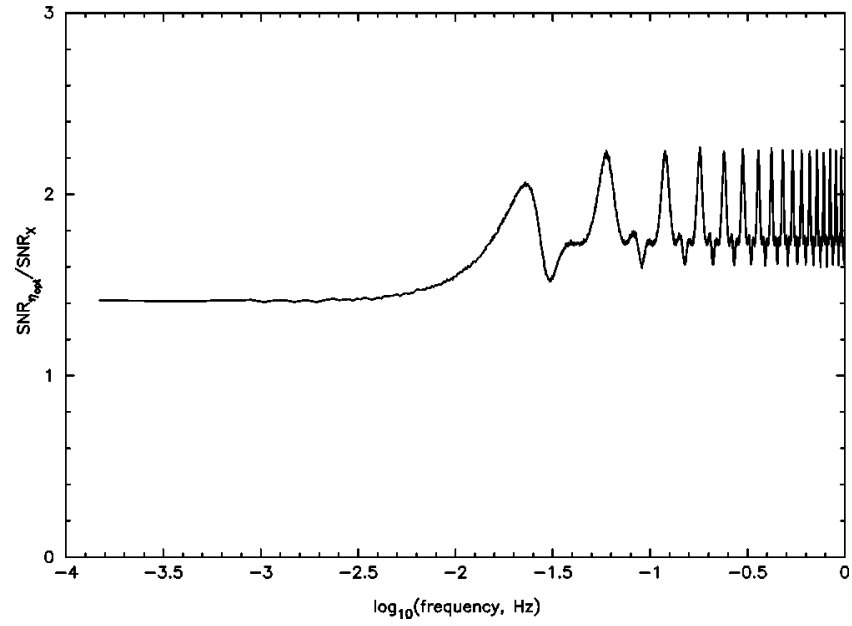

FIG. 4. The optimal SNR divided by the SNR of a single Michelson interferometer, as a function of the Fourier frequency $f$. The sensitivity gain in the low-frequency band is equal to $\sqrt{2}$, while it can get larger than 2 at selected frequencies in the high-frequency region of the accessible band. The integration time has been assumed to be one year, and the proof mass and optical path noise spectra are the nominal ones. See the main body of the paper for a quantitative discussion of this point.

or comparable to the other modes and can dominate the SNR improvement at selected frequencies.

\section{CONCLUSIONS}

The use of Time-Delay Interferometry has shown that LISA has the capability to simultaneously observe gravitational waves in the millihertz band with several, and rather different, interferometric data combinations. In this paper we have identified, for a given (but otherwise arbitrary) gravitational wave signal, the particular interferometric combination that gives maximum signal-to-noise ratio. In this context we have actually shown that LISA should no longer be regarded as a single-instrument mission, but rather as a network of interferometer detectors of gravitational radiation working in coincidence. We have identified the general expression of the optimal combination of the generators $(\alpha, \beta, \gamma)$, which should be used when observing a specified gravitational wave signal.

Under rather general assumptions on the properties of the noise correlation matrix, and for sinusoidal gravitational wave signals that are randomly distributed over the celestial sphere and over the polarization states, we have found that the sensitivity gain of the optimal data combination over that

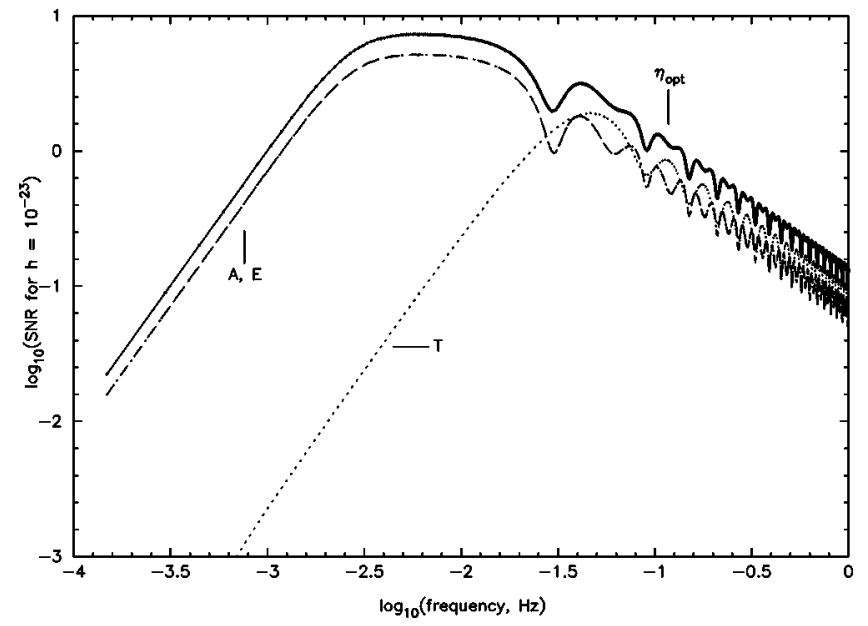

FIG. 5. The SNRs of the three combinations, $(A, E, T)$, and their sum as a function of the Fourier frequency $f$. The SNRs of $A$ and $E$ are equal over the entire frequency band. The SNR of $T$ is significantly smaller than the other two in the low part of the frequency band, while it is comparable to (and at times larger than) the SNR of the other two in the high-frequency region. See text for a complete discussion.

of the Michelson combination can be significant, and it varies over the frequency band accessible by LISA. In the low part of the frequency band such improvement is equal to $\sqrt{2}$, and it grows to values larger than $\sqrt{3}$ at higher frequencies and in small frequency bands centered on frequencies that are integer multiple of the inverse of the one-way light-travel time.

The results derived in this paper will have immediate application to the solution of the so called "Inverse Problem" for LISA, that is to say the determination of the source location and of the wave's two independent amplitudes from the data LISA will be able to generate. We will estimate the accuracies in the determination of the source location and of the wave's amplitudes that the optimal combination of the three interferometric responses $(\alpha, \beta, \gamma)$ derived in this paper will imply. This work is in progress, and will be presented in a follow-up publication.

\section{ACKNOWLEDGMENTS}

We would like to thank Dr. Albert Lazzarini and Dr. Frank B. Estabrook for stimulating conversations while this work was in progress. The research was performed at the Jet Propulsion Laboratory, California Institute of Technology, under contract with the National Aeronautics and Space Administration.
[1] J.W. Armstrong, F.B. Estabrook, and M. Tinto, Astrophys. J. 527, 814 (1999).

[2] F.B. Estabrook, M. Tinto, and J.W. Armstrong, Phys. Rev. D 62, 042002 (2000)

[3] M. Tinto, F.B. Estabrook, and J.W. Armstrong, Phys. Rev. D 65, 082003 (2002).
[4] M. Tinto, J.W. Armstrong, and F.B. Estabrook, Phys. Rev. D 63, 021101(R) (2000).

[5] S.V. Dhurandhar, K.R. Nayak, and J.-Y. Vinet, Phys. Rev. D 65, 102002 (2002).

[6] P. Bender, K. Danzmann, and the LISA Study Team, Laser Interferometer Space Antenna for the Detection of Gravita- 
tional Waves, Pre-Phase, A Report, MPQ233 (Max-PlanckInstitüt für Quantenoptik, Garching, 1998).

[7] B. Noble, Applied Linear Algebra (Prentice-Hall International, Englewood Ciffs, NJ, 1969), p. 378.

[8] S. Selby, Standard of Mathematical Tables (The Chemical Rubber Co., Cleveland, OH, 1964), p. 131.
[9] L.S. Finn, Phys. Rev. D 63, 102001 (2001).

[10] S. Wolfram, MATHEMATICA: User manual, Wolfram Research, Inc., 2002.

[11] M. Tinto, F. B. Estabrook, and J. W. Armstrong, LISA PreProject Publication http://www.srl.caltech.edu/lisa/tdi_wp/ LISA_Whitepaper.pdf, 2002. 